\title{
A Tunable Cavity-Locked Diode Laser Source for Terahertz Photomixing
}

\author{
Shuji Matsuura, Pin Chen, Geoffrey A. Blake, John C. Pearson, and Herbert M. Pickett, Member, IEEE
}

\begin{abstract}
An all solid-state approach to the precise frequency synthesis and control of widely tunable terahertz radiation by differencing continuous-wave diode lasers at $850 \mathrm{~nm}$ is reported in this paper. The difference frequency is synthesized by three fiber-coupled external-cavity laser diodes. Two of the lasers are Pound-Drever-Hall locked to different orders of a Fabry-Perot (FP) cavity, and the third is offset-frequency locked to the second of the cavity-locked lasers using a tunable microwave oscillator. The first cavity-locked laser and the offset-locked laser produce the difference frequency, whose value is accurately determined by the sum of an integer multiple of the free spectral range of the FP cavity and the offset frequency. The dual-frequency $850-\mathrm{nm}$ output of the three laser system is amplified to $500 \mathrm{~mW}$ through two-frequency injection seeding of a single semiconductor tapered optical amplifier. As proof of precision frequency synthesis and control of tunability, the difference frequency is converted into a terahertz wave by optical-heterodyne photomixing in low-temperature-grown GaAs and used for the spectroscopy of simple molecules. The 3-dB spectral power bandwidth of the terahertz radiation is routinely observed to be $\lesssim 1 \mathrm{MHz}$. A simple, but highly accurate, method of obtaining an absolute frequency calibration is proposed and an absolute calibration of $10^{-7}$ demonstrated using the known frequencies of carbon monoxide lines between 0.23-1.27 THz.
\end{abstract}

Index Terms-Calibration, diode lasers, photomixing, source, terahertz waves.

\section{INTRODUCTION}

A DVANCES IN laser technology are facilitating a number of new areas of research in physics and chemistry such as the coherent control of matter and a variety of applications involving high-resolution spectroscopy. The generation of coherent lightwaves in the terahertz or far-infrared frequency region has been investigated by many researchers with limited success and currently forms a frontier in optical science [1]. In general, terahertz frequencies are suitable for the laboratory

\footnotetext{
Manuscript received September 29, 1998; revised December 10, 1999. This work was supported by the National Aeronautics and Space Administration under a contract. The work of G. A. Blake was supported by the National Aeronautics and Space Administration, and by the National Science Foundation.

S. Matsuura was with the Division of Geological and Planetary Sciences, California Institute of Technology, Pasadena, CA 91125 USA (e-mail: matsuura@gps.caltech.edu). He is now with Infrared Astrophysics, Institute of Space and Astronautical Sciences, Kanagawa 229-8510, Japan.

P. Chen was with the Division of Chemistry and Chemical Engineering, California Institute of Technology, Pasadena, CA 91125 USA. He is now with the Time and Frequency Division 847.10, National Institute of Standards and Technology, Boulder, CO 80303 USA.

G. A. Blake is with the Division of Geological and Planetary Sciences, California Institute of Technology, Pasadena, CA 91125 USA.

J. C. Pearson and H. M. Pickett are with the Jet Propulsion Laboratory, California Institute of Technology, Pasadena, CA 91109 USA.

Publisher Item Identifier S 0018-9480(00)02054-8.
}

study of low-energy light-matter interactions, such as phonons in solids, rotational transitions in molecules, vibration-rotation-tunneling behavior in weakly bound clusters, electronic fine structure in atoms, thermal imaging of cold sources, and plasma dynamics. In remote-sensing applications, sources such as interstellar molecular clouds are optically opaque due to the large amounts of cold dust therein. As a result, these objects absorb short wavelength radiation and reradiate most of it in the terahertz region, making terahertz observations of interstellar material in our own galaxy and external galaxies essential for studying the origin and evolution of the universe. Another important remote-sensing application is terahertz studies of the upper reaches of both terrestrial and planetary atmospheres, which also yield critical insights into the nature of atmospheric chemistry and dynamics.

In order to realize the applications described above, various types of terahertz sensors have been proposed and developed for both high- and low-resolution applications. In the area of high spectral resolution detectors, progress in cryogenic heterodyne mixer technology has opened up the possibility of complete coverage of the terahertz spectral region with low-noise heterodyne receivers should suitable local oscillators (LO's) be developed. In the area of sources suitable for LO's, molecular gas lasers are available at moderate power and discrete frequencies throughout the region, and electron beam tubes such as backward-wave oscillators have been demonstrated to $1.5 \mathrm{THz}$ with milliwatt output powers and $\sim 10 \%$ bandwidth. P-type germanium lasers also provide milliwatt output power and reasonable tuning. Free-electron lasers can cover the entire terahertz spectral region and provide very high power. These fundamental oscillators are, at present, massive (from the standpoint of remote-sensing platforms) and suffer from pulsed operation in general, but research efforts are underway to reduce their size and enable $\mathrm{CW}$ operation. Alternatively, harmonic up-conversion from phase-locked microwave sources and down-conversion from optical sources into the terahertz region now offer excellent frequency control and CW operation. However, to date, both these approaches have suffered from poor conversion efficiency and the low output power that results.

Optical heterodyne mixing (photomixing) in voltage-biased low-temperature-grown (LTG) GaAs photoconductors with planar terahertz antennas has recently become an attractive frequency down-conversion method because it has demonstrated relatively high conversion efficiency [2], [3]. An advantage of the down-conversion technique over harmonic generation is that the terahertz source produced by application of optical/near-infrared (IR) lasers onto a semiconductor photomixer is widely tunable. Diode-laser-based systems are particularly 


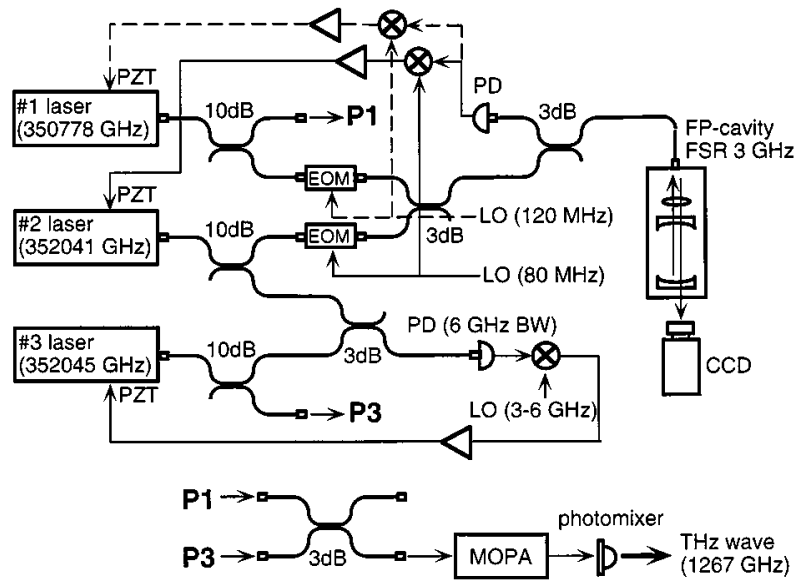

Fig. 1. Schematic diagram of the three DBR laser system that synthesizes a precise difference frequency. The indicated laser frequencies represent the actual values to generate the terahertz wave at $1.267 \mathrm{THz}$.

promising in this type of application since they combine low power consumption and long lifetime in an inexpensive and compact package [4], [5]. Such systems have already been applied to laboratory spectroscopy by several authors [6]-[8]; but the frequency accuracy of the terahertz-wave output obtained in previous studies has not been sufficient for high-resolution laboratory spectroscopy or heterodyne remote-sensing applications.

In this paper, we present a method for synthesizing a near optical difference frequency with the precision necessary for high-resolution terahertz applications. In order to demonstrate the system capability, the difference frequency was turned into a terahertz wave with an LTG-GaAs photomixer and used for spectroscopy. Since the output power of the difference frequency synthesizing laser is marginal or not sufficient to efficiently generate terahertz radiation, the optical radiation is amplified through the master-oscillator power-amplifier (MOPA) technique in the final stage of the laser system. The MOPA provides sufficient power to take full advantage of the quadratic dependence of the photomixing process on pump laser power [2] or to drive difference frequency generation in any good nonlinear optical materials should they become available. It should be further noted that this method of difference frequency synthesis can be used at any optical or infrared wavelength where suitable lasers and optics exist.

\section{LASER SySTEM DESIGN AND PERFORMANCE}

\section{A. General Concept}

The light source of the difference frequency system consists of three diode lasers, as is depicted in Fig. 1. Each laser diode is outfitted with a collimating lens, a length tunable externalcavity assembly, and the necessary optics to circularize and fiber couple the optical radiation. Alignment is maintained by a compact aluminum rail structure. All the optical signal processing components are implemented in polarization-maintaining (PM) single-mode fiber. The optical components used and their layout is shown in Fig. 1. Commercial components were used whenever available, but some homemade components were necessary. The fiber optics offer flexibility, compactness, insensitivity to vibration, ease of optical alignment, and eye protection. The optical fiber also serves as a spatial filter, allowing two different laser frequencies to be combined with nearly perfect spatial mode overlap. The latter is critical in the Fabry-Perot (FP) etalon alignment, in achieving equal amplification in the final MOPA stage, and in efficient photomixer operation. The major drawbacks of the fiber-based optical system as opposed to a free-space approach are the increased cost and the optical insertion losses of the PM fiber. Due to the coupling losses, the output power of the present laser system was insufficient to optimally pump the photomixer used to generate terahertz waves. As a result, a tapered amplifier was employed as the final optical element before the photomixer once it had been determined that the spectral properties of the seed lasers were preserved in the amplification process [14].

Frequency control is achieved by locking two of the lasers (\#1 and \#2) to different longitudinal modes of an ultra-low-expansion (ULE) FP etalon. The difference frequency between the two cavity-locked lasers is discretely tunable in steps of the cavity free spectral range (FSR). The third laser (\#3) is heterodyne phase locked to one of the cavity-locked lasers (\#2) with a tunable 3-6-GHz microwave synthesizer. The difference frequency between the \#1 and \#3 lasers is determined by the sum of integral multiples of the FSR ( $3 \mathrm{GHz}$ in the present system) of the reference cavity and the microwave offset frequency. The accuracy of the difference frequency is determined by the accuracy of the FSR measurement along with any dc offset in the electrical portions of the lock loops. The microwave offset frequency is locked to a high accuracy ( 1 in $\left.10^{-12}\right)$ reference source and measured by a counter locked to the same reference in order to correct, in real time, any electrical offset in that lock loop. The ULE material has a thermal expansion coefficient at room temperature of $\alpha=-2 \times 10^{-10}{ }^{\circ} \mathrm{C}^{-1}$, which is comparable to the stability of a good quartz reference oscillator in conventional microwave sources. As will be described later, the measurement of molecular transitions allows any dc offsets in the two cavity-locked loops to be determined and the system to be calibrated to very high precision. The optical and electrical details of all the major subsystems of this terahertz difference frequency generator are described in detail in the next few sections.

\section{B. Laser Assembly and Control}

The full schematic of the external-cavity diode laser assembly is presented in Fig. 2. The assembly consists of an SDL5722 852-nm 150-mW distributed Bragg-reflector (DBR) diode laser, an $f=4.5 \mathrm{~mm}$ collimating lens, an external cavity comprised of a $4 \%$ partial reflector mounted on a piezoelectric transducer (PZT), an anamorphic prism pair, a 60-dB optical isolator, an $f=8 \mathrm{~mm}$ optic, and a adjustment flexure for fiber coupling. The actual external cavity is formed by a roof-top mirror glued onto a $20 \%$ beam-splitter cube. The edge of the roof-top mirror is oriented along the short axis of the elliptical beam from the DBR laser, which is more sensitive to the tilt angle than is the long axis. As such, the round-trip mirror configuration offers relatively alignment-free optical feedback. The reflector and DBR laser chip constitute an external cavity with a length of approximately $50 \mathrm{~mm}$. The $3: 1$ aspect ratio 


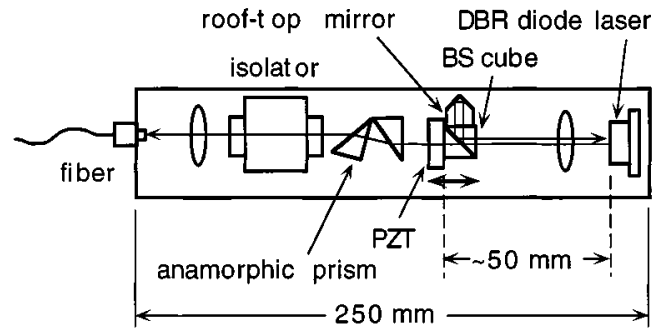

Fig. 2. Schematic diagram of the external-cavity DBR laser assembly.

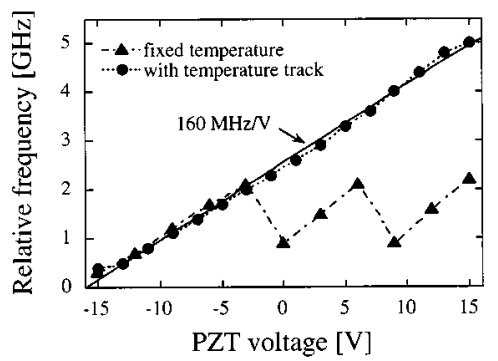

Fig. 3. Frequency of the external-cavity laser plotted against the PZT control voltage. The triangles represent the tuning curve during which the temperature of the DBR laser is fixed, and the circles represent that with temperature tracking.

beam transmitted through the partial reflector is circularized by the anamorphic prism pair to achieve better spatial overlap with the PM fiber and thereby improve the coupling efficiency. These components are assembled in a $250-\mathrm{mm}$-long aluminum rail to maintain alignment. The typical optical power coupled into the PM fiber was approximately $30 \%$ of the original power emitted from the DBR laser. The transmission of the free-space optics in the laser assembly was measured at $\sim 70 \%$, while the fiber-coupling efficiency was typically $-50 \%$. The theoretical maximum fiber-coupling efficiency calculated from the measured beam size and the fiber core size is $60 \%$ of the DBR power. The best coupling ever achieved by this optical system was $55 \%$. Other power losses include the use of a passive 3-dB directional coupler to combine the output laser beams and $\sim 1-\mathrm{dB}$ losses at each fiber connector. As a result, the typical power available at the fiber output port is $\sim 30 \mathrm{~mW}$. The difference in the typical and maximum powers is due to slight thermal deformations in the rail assembly and the slow buildup of dust and dirt in the fiber connectors over time.

The DBR diode laser coupled to the external-cavity oscillates at the cavity mode that is the closest to the gain maximum of the DBR laser. The FSR of the external cavity was $\sim 3 \mathrm{GHz}$, corresponding to the separation between the partial reflector and DBR chip. The laser frequency is continuously tunable since the external cavity's FSR can be tuned by the PZT at the rate of $160 \mathrm{MHz} / \mathrm{V}$. The continuous PZT tuning range is limited by mode hops to different transverse external cavity modes favored by the gain profile of the DBR laser. This effect, for a DBR laser operating at fixed temperature and current, is shown in Fig. 3 by the triangles. In order to avoid such mode hops, the laser's temperature can be adjusted to maximize its gain at the external-cavity mode frequency, completely suppressing the mode hops and facilitating continuous tuning over the PZT tuning limit of $5 \mathrm{GHz}$ (which corresponds to $\pm 15 \mathrm{~V}$ ), as shown by the circles in Fig. 3. Coarse frequency tuning spanning $\gtrsim 700$ $\mathrm{GHz}$ is available by changing the laser temperature over the range from $5{ }^{\circ} \mathrm{C}$ to $30{ }^{\circ} \mathrm{C}$ (or $27 \mathrm{GHz} / \mathrm{K}$ ). Although the injection current also affects the laser frequency ( $\gtrsim 600 \mathrm{MHz} / \mathrm{mA})$, the current was normally set to be constant unless fine or fast frequency tuning was necessary, as is described in Section III.

Stable diode laser operation requires that both the injection current and the temperature be precisely controlled. A low-cost high-performance control circuit has been designed in order to satisfy the control criteria. The current controller was adapted from Libbrecht and Hall's work [9] to suit the anode-ground configuration of the DBR lasers. The current is controlled by comparing a reference voltage to a voltage drop produced by the laser current through a series resistor. The circuit components of this current source were carefully chosen to maximize the dc and thermal stability. The voltage reference was an Analog Devices 584L (temperature coefficient $3 \mathrm{ppm} /{ }^{\circ} \mathrm{C}$ ), while the sense resistor was a 33.3- $\Omega$ four-lead Vishey resistor (temperature coefficient $0.5 \mathrm{ppm} /{ }^{\circ} \mathrm{C}$ ). The sensing circuit was designed with filters to eliminate response above $10 \mathrm{kHz}$ and with lownoise low-offset voltage op-amps (Analog Devices 784B) to drive a field-effect transistor (FET) (IRFD-120) that provides the output current of 0-300 mA. An average current noise of 7 $\mathrm{nA} / \mathrm{Hz}^{1 / 2}$ over the range of $5-2000 \mathrm{~Hz}$ at $150-\mathrm{mA}$ dc current has been measured. The noise declines to immeasurable levels by $10 \mathrm{kHz}$. The laser temperature is stabilized by a bridge circuit incorporating both differential drive and detection using the DBR laser's built in thermistor and thermo-electric cooler. The thermal time constant of a DBR laser mounted in the aluminum rail is $24 \mathrm{~s}$, but the temperature control circuit achieves a loop bandwidth of $12 \mathrm{~Hz}$. The temperature can be set in 16-bit steps from $3{ }^{\circ} \mathrm{C}$ to $30{ }^{\circ} \mathrm{C}$ by a serial-controlled digital/analog (D/A) converter. As with the current controller, careful selection of low-noise circuit components was essential, and a temperature stability of better than $1 \mathrm{mK}$ has been achieved. The observed long-term unlocked frequency stability $(1 \mathrm{~h})$ of the DBR is 300 $\mathrm{MHz}$. The current supply, temperature controller, and PZT controller, including the frequency stabilization circuit, are fabricated on a 4 in $\times 6$ in circuit board that is connected to a computer through a serial interface for monitor and control purposes. This single board design has the advantages of compactness, high performance, and low cost.

In spite of the much lower level of optical feedback than that of conventional external-cavity diode lasers, a significant narrowing of the laser linewidth was realized. The linewidth measured by the delay-line self-heterodyne technique was narrower than $500-\mathrm{kHz}$ full width half at maximum (FWHM), which is near the spectral resolution limit imposed by the optical delay length of $200 \mathrm{~m}$. This linewidth is a significant improvement over the free-running DBR linewidth of several to several tens of megahertz. According to a previous study on the frequency stability of diode lasers at various feedback levels [10], our case falls into the weak feedback regime where stable single-mode narrow-line oscillation should be observed. If the feedback level is higher than this regime, but still lower than the original laser chip facet's feedback, the oscillation becomes unstable and the 


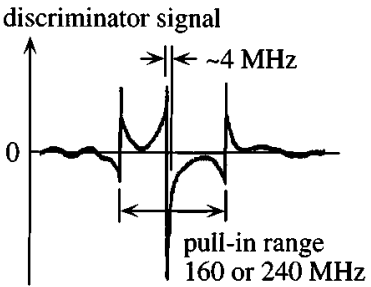

Fig. 4. Dispersion signal of the cavity fringe used for frequency stabilization.

coherence collapse results in a drastic line broadening. This effect has been observed at a feedback level of $\sim 20 \%$ with the same optical configuration. If much more intense feedback is applied or if the laser chip facet is AR-coated, stable narrow-line oscillation in a strong feedback regime could again be obtained with somewhat improved linewidths, but such intense feedback would impose a steep cost on the available output. It should be noted that the optical feedback in this system is electrically equivalent to a very fast feedback circuit. The reasons for using optical feedback instead of fast electrical feedback will be addressed in the following section.

\section{Frequency Stabilization}

The P1 and P2 lasers were cross polarized in order to allow them to be locked to different longitudinal modes of the ULE etalon by the Pound-Drever-Hall method [11], [12]. FM sidebands were generated on the two cavity-locked lasers with electrooptic phase modulators (EOM) operating at 80 and $120 \mathrm{MHz}$, respectively. The modulation index was chosen to maximize the phase shift seen in the reflection from the cavity. To verify coupling to the fundamental longitudinal cavity mode, the transmitted beam profile was monitored by a charge-coupled device $(C C D)$ camera. Since density and humidity changes in air can cause FSR fluctuations through refractive index changes, the cavity was installed in a sealed box, which was evacuated and then back filled with dry nitrogen so that the density inside the box was constant. The phase of the beam reflected from the cavity was compared to that of the modulation in a frequency multiplier (Analog Devices 834Q). When the laser frequency is within the modulation frequency of the cavity resonance, the output of the frequency multiplier provides a dc frequency dispersion, which crosses zero at the cavity resonance. Fig. 4 shows the dispersion curve at the mixer output obtained by sweeping the laser frequency through the cavity resonance. The central linear portion of the dispersion curve centered at the cavity is used to generate an error signal voltage, which is fed back to the PZT of the external laser cavity with a simple servo electronic circuit consisting of an integrator and an amplifier. The linear part of frequency discriminator shown in Fig. 4 is $\sim 4 \mathrm{MHz}$, in accordance with the FSR of the cavity of $3 \mathrm{GHz}$ and its finesse of 750. As can be seen in Fig. 4, the pull-in range of this locking method is limited by the distance to the upper and lower sidebands, or two times modulation frequency (160 and $240 \mathrm{MHz}$ ). In the present laser system, the loop bandwidth of the PZT control circuit was limited to $3 \mathrm{kHz}$ to avoid acoustic resonances in the support structure of the partial reflector and the PZT. It was observed that only $200 \mathrm{~Hz}$ of bandwidth was required for stabilization, but the additional bandwidth was retained to facilitate sweeping of the offset laser.

The offset-locked laser is stabilized to a beat note signal between the \#2 and \#3 lasers detected by a 6-GHz bandwidth photodetector and compared to tunable frequency generated by a microwave synthesizer. In order to implement a phase lock with the same electronics as the cavity lock, the signal was split with one arm sent through a delay line, and the resulting phase shift between the two signals was used to generate the error signal for the PZT of the \#3 laser. The delay line has an effective reference frequency of $71.9 \mathrm{MHz}$. However, concerns about the accuracy and stability of this effective lock frequency led to the use of a counter. The offset frequency is measured precisely by a microwave counter locked to a high-precision reference, making any drifts or offsets in the phase-locked scheme irrelevant to the system calibration. The offset frequency can be continuously tuned over $5 \mathrm{GHz}$ by stepping the synthesizer frequency and tracking the PZT voltage. The maximum sweep rate of $\lesssim 100 \mathrm{MHz} / \mathrm{s}$ is limited by the feedback loop bandwidth.

The overall system performance was assessed by observing a beat note between the \#1 and \#3 lasers with a $25-\mathrm{GHz}$ bandwidth photodetector and a spectrum analyzer. Fig. 5 shows a 12-GHz beat spectrum for a 1-s integration time and a spectral resolution of $100 \mathrm{kHz}$. The FWHM spectral power bandwidth is approximately $800 \mathrm{kHz}$ and much narrower than that of the previous laser systems for the photomixing, i.e., 2-5 MHz [6], [8]. This result confirms the self-heterodyne linewidth measurements of $\sim 500 \mathrm{kHz}$. The short-term linewidth of each laser is determined entirely by the optical feedback from the external cavity because the 3-kHz bandwidth of the locked loop circuit is much less than the laser linewidth. Short-term $(50 \mathrm{~ms})$ DBR linewidths of $\sim 50 \mathrm{kHz}$ have been achieved by weak optical feedback from FP cavities [7]. The additional narrowing is due to a $180^{\circ}$ phase shift over a narrow $(60 \mathrm{MHz})$ cavity width compared to the same phase shift over $1.5 \mathrm{GHz}$ in the scheme presented here. Fast electronic feedback to the laser current can also be used to narrow the laser linewidths [13], but the DBR lasers used here have some unfortunate characteristics that made this impractical.

Indeed, stabilization and narrowing of 850-nm DBR lasers with electrical feedback was attempted in the first design iteration of this laser system. The DBR lasers have two means of modulation. The first is ohmic heating of the active region by the injected current. Since the active region of the laser is fairly small, this process is fairly fast and has a 3-dB knee near $600 \mathrm{kHz}$. The tuning rate is $580 \mathrm{MHz} / \mathrm{mA}$ when the diode chip is under active temperature control. The other effect is a change in the index of refraction due to the electrons injected into the active region. This effect is almost instantaneous (many gigahertz of bandwidth), and has a magnitude of only $10 \%$ of the thermal effect, from which it is $180^{\circ}$ out of phase. Due to the differing magnitudes of the two modulation effects, there is no null in the modulation response, but there is a $180^{\circ}$ phase shift in the modulation response once $1.2 \mathrm{MHz}$ is reached. Unfortunately, this is considerably slower than the observed natural linewidth of 3-10-MHz FWHM, depending on where in relation to the ideal current the laser was operating. It was observed that the 


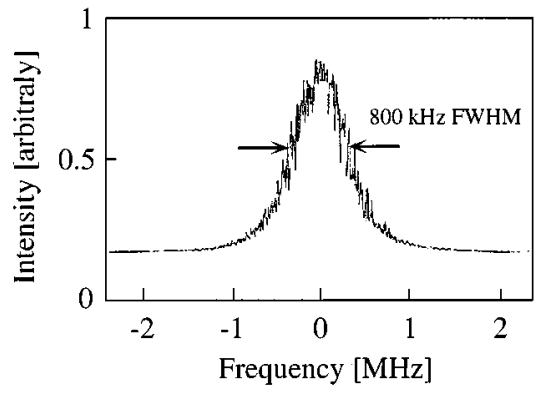

Fig. 5. Spectrum of the beat note signal between the \#1 and \#3 lasers.

center frequency of the DBR lasers could be stabilized with current feedback and less than $10 \mathrm{kHz}$ of locked loop bandwidth, but no linear locked loop fully encompassing the $1.2-\mathrm{MHz} 180^{\circ}$ phase-shift point could be developed to narrow the laser output. As a result of these unfortunate phase shifts, optical feedback has been adopted as the preferred method of narrowing a DBR laser. A combination of optical and electrical feedback may ultimately prove to be the best method of narrowing the DBR laser; however, this has not been attempted at this time.

One problem experienced in the system design was the relatively large temperature changes in our laboratory and the resulting changes of temperature equilibrium in the external cavity and laser themselves. The PZT voltage generally compensates for these drifts, but they were on many occasions large enough to exceed the PZT error signal limit. This, coupled with the DBR laser's propensity to wake up in one of two modes separated by approximately $30 \mathrm{GHz}$, led to active stabilization of the temperature of the laser rails. Once this was done, all-day-long cavity locks of the laser have been routinely achieved without adjusting the temperature of the laser itself. Additionally, with the active temperature stabilization, the DBR lasers routinely turn on and equilibrate to within a few megahertz of where they were last used. The long-term frequency stability of the system when locked to the ULE cavity is as good as our ability to determine the center of the $500-\mathrm{kHz}$ linewidth and it is has been repeatable to that accuracy for a period of several months.

\section{Dual-Frequency MOPA Operation}

Since the maximum output power of the fiber-coupled laser system is $\sim 30 \mathrm{~mW}$ and the optical alignment was critical to provide such power levels, it was marginal or not sufficient to efficiently generate terahertz radiation by photomixing. In order to provide a margin in the output power, we amplified the dual-frequency signal with a single traveling-wave 850-nm semiconductor tapered optical amplifier, which was the central component of a commercial external-cavity single-mode laser (SDL8630). A detailed description of the dual-frequency MOPA system and its properties has been given elsewhere [14].

The output power of the MOPA was $500 \mathrm{~mW}$ and constant to within 5\% over the entire range of the difference frequency $(\sim 1.3 \mathrm{THz})$. The injection seeded bandwidth of such amplifiers is of order 20-25 nm [15], and two frequency amplification should, therefore, be feasible to offsets of some $10-15 \mathrm{THz}$ with appropriate seed lasers. The power ratio between the amplified two frequency components was close to unity over a wide range of difference frequencies, specifically from $\sim 10 \mathrm{GHz}$ to 1.3 THz. Unbalanced amplification between the two frequencies caused by a nonlinear gain effect occurred only at difference frequencies lower than $10 \mathrm{GHz}$ [14]. The spectrum of beat signal between the two frequency outputs was identical to that of the master laser as long as the difference frequency was greater than $10 \mathrm{GHz}$.

Dual-frequency MOPA operation has the advantages of high power output and guarantees excellent spatial overlap of the two frequency beams, which is essential for efficient opticalheterodyne conversion. The available maximum output power of previous diode laser sources for photomixing was less than $100 \mathrm{~mW}$, while the present MOPA system provides 500-mW output. Unfortunately, since the maximum pump laser power which the small active area LTG-GaAs photomixers used in this study can handle is limited to approximately $50 \mathrm{~mW}$ by their thermal failure [16], we had to attenuate the MOPA output appropriately. As a result, the terahertz output power is limited to sub- $\mu \mathrm{W}$ levels. The development of large-area traveling-wave photomixers, which can handle laser powers well in excess of $500 \mathrm{~mW}$, will allow us to take full advantage of high power of the MOPA [17]. Further, the MOPA is useful not only for photomixing in the LTG GaAs, but also for difference-frequency generation with nonlinear optical media that have much higher damage thresholds than LTG GaAs.

\section{TERAHERTZ-WAVE GENERATION}

As described in Section I, the dual-frequency laser system can generate terahertz waves by difference frequency mixing in LTG-GaAs ultra-fast photoconductors or other nonlinear optical media. Here, we demonstrate the performance of the laser system with high-resolution rotational spectroscopy of the simple molecules acetonitrile $\left(\mathrm{CH}_{3} \mathrm{CN}\right)$ and carbon monoxide (CO). Due to the lack of spectral-analysis techniques in the terahertz region, spectroscopic measurements provide one of the best diagnosis of frequency and spectral purity, which is essential if this source is going to be useful as a LO.

\section{A. Spectroscopy}

The LTG-GaAs photomixer used in the present experiment was grown on a semiinsulating GaAs substrate, and a planar log-spiral antenna with $0.2-\mu \mathrm{m}$ interdigitated electrodes and 1.8- $\mu \mathrm{m}$ gaps in a $8 \mu \times 8 \mu \mathrm{m}$ active area was etched on the wafer [16]. The photomixer was mounted on the flat surface of a 10-mm-diameter hyper-hemispherical lens made of high-resistivity silicon. Most of the radiation generated by the antenna and electrodes passes into the photomixer substrate and through the Si lens. A dc-bias voltage of $20 \mathrm{~V}$ was applied to the electrodes by a constant current supply set at $0.5 \mathrm{~mA}$. The output power of the MOPA was attenuated to $30 \mathrm{~mW}$ in order to keep the input power to the photomixer well below the thermal failure threshold. Under these conditions, the photomixer provided a maximum output power of $\sim 0.1 \mu \mathrm{W}$ at $1 \mathrm{THz}$, which is similar to the values obtained in previous reports [3], [6]-[8], [16]. The spectral bandwidth of the generated terahertz waves was approximately $700 \mathrm{GHz}$, in accordance with the carrier lifetime of the LTG GaAs of $\tau \sim 200-300$ fs and the 


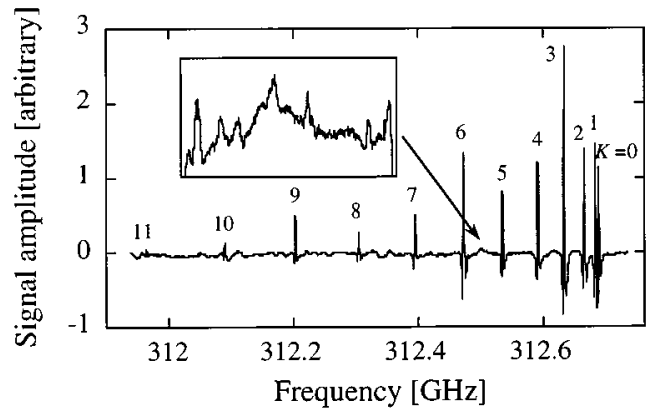

Fig. 6. Second-derivative absorption spectrum of $\mathrm{CH}_{3} \mathrm{CN} \mathrm{J}_{K}=16_{K} \rightarrow 17_{K}$ rotational transitions near $312 \mathrm{GHz}$. The inset is expanded view of the ${ }^{13} \mathrm{C}$ isotopic features.

photomixer $R C$ time constant, where $R=72 \Omega$ is the radiation impedance and $C=0.5 \mathrm{fF}$ is the electrode capacitance [16].

The terahertz-wave output beam from the photomixer was collimated with a combination of the silicon hyper-hemispherical lens and a Teflon lens. The collimated beam was passed through an 8-cm-long 1-in-diameter gas cell fitted with polyethylene windows for absorption spectroscopy. The beam transmitted through the cell was weakly focused with a Teflon lens and fed into a $4.2-\mathrm{K}$ InSb hot-electron bolometer or a $1.8-\mathrm{K}$ composite Si bolometer. The tone-burst modulation [18] was used to obtain the absorption spectra of various molecules. A major advantage of the tone burst method for terahertz spectroscopy, as compared to traditional FM modulation, is that sensitive detection with slow detectors such as silicon composite bolometers can be achieved. The injection current of the \#1 cavity-locked laser was modulated with a $2-\mathrm{MHz}$ tone, above the cavity-locked-loop bandwidth, at a $10-\mathrm{kHz}$ burst rate. A lock-in amplifier, detecting at $10 \mathrm{kHz}$, was used to demodulate the detector signal generating the traditional second derivative of molecular absorption features. Since the modulated laser is different from the swept laser, power variations in the swept laser are not modulated.

Fig. 6 presents the absorption spectrum of the $\mathrm{CH}_{3} \mathrm{CN} \mathrm{J}_{K}=$ $16_{K} \rightarrow 17_{K}$ rotational transitions near $312 \mathrm{GHz}$. The spectrum was taken with a sweep rate of $2 \mathrm{MHz} / \mathrm{s}$, and is plotted as a function of the microwave offset frequency. The data were recorded at seven sample/s with a lock-in amplifier. The spectrum shows the well known $K$-structure of a symmetric top, with $K$ components from $K=0-11$ assigned in the spectrum. The $K=0,1$ lines, which are separated by $\sim 6 \mathrm{MHz}$, are clearly resolved. The gas pressure was $60 \mathrm{mtorr}$, and the observed linewidths are consistent with a convolution of pressure broadened linewidths and the instrument response. The minimum detectable absorption of this system is estimated to be $\sim 10^{-5}$, and is detector noise limited. As shown in the inset of Fig. 6, the spectral lines of the ${ }^{13} \mathrm{C}$-isotopomer of acetonitrile, which has a natural abundance of roughly $1 \%$, could be also detected with a signal-to-noise ratio of $\sim 20$, consistent with the detection limit. These results indicate that the spectral purity, frequency control, and output power of this system is sufficient for the laboratory spectroscopic study of molecules at terahertz frequencies, as well as many LO applications.
TABLE I

MEASuRed AND CALCulated CO ROTATIONAL FREQUENCIES

\begin{tabular}{rccc}
\hline \hline$J$ & Calculated $[\mathrm{MHz}]$ & Measured $[\mathrm{MHz}]$ & difference $[\mathrm{MHz}]$ \\
\hline 1 & 230538.00 & 230537.88 & -0.12 \\
2 & 345795.98 & 345795.95 & -0.03 \\
3 & 461040.75 & 461040.90 & 0.15 \\
4 & 576267.91 & 576268.03 & 0.12 \\
5 & 691473.05 & 691473.06 & 0.01 \\
6 & 806651.77 & 806651.60 & -0.17 \\
7 & 921799.67 & 921799.85 & 0.18 \\
8 & 1036912.35 & 1036912.80 & 0.45 \\
9 & 1151985.40 & 1151985.34 & -0.06 \\
10 & 1267014.44 & 1267014.10 & -0.34 \\
\hline
\end{tabular}

\section{B. Frequency Calibration}

For further spectroscopic measurements, such as the search for unknown molecular lines and for use in astronomical observations, absolute frequency calibration of the difference frequency is necessary. Since the accuracy of the difference frequency is defined by the reference FP cavity, the calibration must include a precise measurement of the FSR of the cavity. Once the exact value of the FSR is obtained, the difference frequency can be determined, in principle, to within an accuracy of $\sim 10^{-10}$, if temperature fluctuations of the cavity are kept below $\sim 1 \mathrm{C}$, because of the extremely low thermal expansion coefficient of the ULE material. Well-known molecular lines in the terahertz region, such as the rotational transitions of carbon monoxide (CO), are suitable for accurate calibration since the frequencies of these terahertz molecular transitions correspond to 2300 times the FSR and can be easily measured to within an accuracy of $10^{-7}$. A number of measurements and the careful use of statistics should allow for a rapid calibration of $10^{-8}$. Other FSR measurements have been proposed and demonstrated to better than $10^{-10}$ [19], but these require the use of expensive fast EOM's matched to the cavity FSR.

Pure rotational transitions of $\mathrm{CO}$ were measured using the same configuration as the acetonitrile measurements. According to the conventional model for the diatomic ${ }^{12} \mathrm{C}^{16} \mathrm{O}$ molecule, the rotational transition lines should appear at frequencies of $\nu=\left(W_{J+1}-W_{J}\right) / h$, and $W_{J} / h=B J(J+1)-D J^{2}(J+$ $1)^{2}+H J^{3}(J+1)^{3}$, where $B=57635.9660 \mathrm{MHz}, D=$ $0.1835053 \mathrm{MHz}, H=1.731 \times 10^{-7} \mathrm{MHz}, h$ is the Planck constant, and $J$ is an integer [20]. Absorption measurements for CO lines with $\mathrm{J}_{\text {lower }}=1-10$ over the range of $230-1267 \mathrm{GHz}$ were carried out by measuring the microwave offset frequency $\nu_{\text {offset }}$ and counting the number of cavity orders between the two cavity-locked lasers. The line position was determined by fitting a parabola to the center of the second derivative line profile. The measured and calculated rotational frequencies and their differences are summarized in Table I. The final measured frequencies were obtained using the calibrated FSR value of the cavity as follows. The cavity FSR for each CO line is simply calculated by dividing $\nu-\nu_{\text {offset }}$ by the cavity-order difference because the dc offset of the difference frequency caused by the dc offset voltage of the locked loop circuit was statistically insignificant. From this data set, the average of the FSR value for all CO line measurements was determined to be 2996757.48 


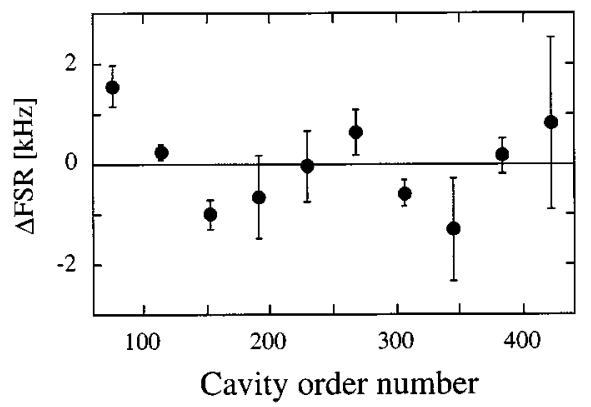

Fig. 7. Result of the FSR measurements by CO lines for $\mathrm{J}_{\text {lower }}=1-10$. The deviation of the FSR values from their average are shown as a function of the cavity-order difference.

$\pm 0.10 \mathrm{kHz}$. Fig. 7 shows the deviation of the FSR values from their average as a function of the cavity-order difference. Even if the scatter of the data around the average value is real, the frequency dependence of the FSR over a 1.3-THz span is constant to within $1 \mathrm{kHz}$. The absolute frequency accuracy of $\lesssim 10^{-7}$ is at least one order of magnitude better than that obtained in previous work [6]-[8]. In this approach, the frequency accuracy is limited by our ability to determine the center of the line profiles, which, in turn, ultimately depends on the instrumental resolution and the signal-to-noise ratio of the spectrum. It should be noted that the FSR could be a function of cavity order due to dispersion of the refractive index of the cavity coatings. However, no variations are convincingly observed and the expected effect is smaller than the current precision [19].

\section{CONCLUSIONS}

The photomixer used in the experiments reported here provides a maximum output power of approximately $0.1 \mu \mathrm{W}$ at $1 \mathrm{THz}$ for a pump laser power of $30 \mathrm{~mW}$. A straightforward extrapolation of the quadratic dependence of the terahertz-wave power on the laser power leads to the prediction that some $10 \mu \mathrm{W}$ of power should be obtainable using the present laser system, whose maximum $850-\mathrm{nm}$ power is $500 \mathrm{~mW}$. However, the maximum pump laser power is currently limited to approximately $50 \mathrm{~mW}$ by thermal failure of the photomixer [16], and the output power measured in previous work has, therefore, been limited to levels of $\lesssim 1 \mu \mathrm{W}$ [3], [6]-[8], [16]. To solve this thermal problem, photomixers with distributed electrode structures and higher thermal conductivity substrates are being developed [16], [17], [21]. Such photomixers can be driven at the full output of the high-power laser system reported here, and will ultimately produce power levels of nearly $10 \mu \mathrm{W}$ at $1 \mathrm{THz}$. Larger frequency offsets are easily obtained with additional DBR lasers or by using grating-tuned external cavity diode lasers [15]. The current power levels are already sufficient for spectroscopic applications in the 3-6-THz region if more sensitive detectors than those available here are used (e.g., $\mathrm{Ga}$ :Ge photoconductors or superconducting microbolometers coupled to planar antennae).

The three laser difference frequency generation and control method presented here is quite general and could be extended to a large number of different lasers. The absolute calibration method is also quite general and can be widely employed, as long a method of generating a terahertz-wave output is available. This frequency control technique is especially important in the $>1-2$ terahertz region, where comparison to a harmonically up-converted frequency reference may be difficult or impossible. The use of an MOPA as a dual-frequency amplifier should facilitate the use of this control method with the next generation of photomixers based on nonlinear optical media such as $\mathrm{LiNO}_{3}, \mathrm{GaP}, \mathrm{GaAs}$, and quantum-well materials. At optical source frequencies, down-conversion methods using nonlinear optical materials might be more efficient than electrooptical down-conversion with photoconductors because the efficiency of the optical down-conversion, in general, has a $\nu^{4}$ dependence in the long-wavelength limit [22]. The present laser system design provides the necessary control to take advantage of any improvements in conversion efficiency using such methods. Further development of nonlinear optical materials and novel devices with large $\chi^{2}$ at diode laser frequencies is also expected in the near future, making precision difference frequency generation essential for their use as terahertz sources.

Finally, the type of terahertz source demonstrated here should be useful not only for terahertz spectroscopy but also as LO's for future airborne and space-borne terahertz heterodyne receivers to be used in atmospheric science and astronomy. The advantage of space-borne telescopes in the terahertz range is their continuous wide-frequency coverage, which is prevented by strong atmospheric water line absorption at low altitudes. The wide tunability of a photomixer-based LO system will make it possible to construct highly tunable heterodyne receivers with a single LO and single mixer. Properties of the current laser system such as its small size, low power consumption, and fiber-connectorized optics also make it highly suitable for space-borne instruments. The development of such remote-sensing terahertz spectrometers is currently in progress.

\section{ACKNOWLEDGMENT}

The authors would like to thank S. Verghese and K. A. McIntosh, MIT Lincoln Laboratory, Cambridge, for preparing the LTG-GaAs photomixers. The authors also thank T. J. Crawford, Jet Propulsion Laboratory, California Institute of Technology, Pasadena, CA, for his technical support. Portions of this work were performed at the Jet Propulsion Laboratory.

\section{REFERENCES}

[1] "Metrology issues in terahertz physics and technology," NIST, vol. 5701, p. 103, 1995.

[2] E. R. Brown, F. W. Smith, and K. A. McIntosh, "Coherent millimeter-wave generation by heterodyne conversion in low-temperature-grown GaAs photoconductors," J. Appl. Phys., vol. 73, pp. 1480-1484, 1993.

[3] E. R. Brown, K. A. McIntosh, K. B. Nichols, and C. L. Dennis, "Photomixing up to $3.8 \mathrm{THz}$ in low-temperature-grown GaAs," Appl. Phys. Lett., vol. 66, pp. 285-287, 1995.

[4] K. A. McIntosh, E. R. Brown, K. B. Nichols, O. B. McMahon, W. F. DiNatale, and T. M. Lyszczarz, "Terahertz photomixing with diode-lasers in low-temperature-grown GaAs," Appl. Phys. Lett., vol. 67, pp. 3844-3846, 1995.

[5] S. Matsuura, M. Tani, and K. Sakai, "Generation of coherent terahertz radiation by photomixing in dipole photoconductive antennas," Appl. Phys. Lett., vol. 70, pp. 559-561, 1997.

[6] A. S. Pine, R. D. Suenram, E. R. Brown, and K. A. McIntosh, "A terahertz photomixing spectrometer-Application to $\mathrm{SO}_{2}$ self-broadening," J. Mol. Spectrosc., vol. 175, pp. 37-47, 1996. 
[7] P. Chen, G. A. Blake, M. C. Gaidis, E. R. Brown, K. A. McIntosh, S. Y. Chou, M. I. Nathan, and F. Williamson, "Spectroscopic applications and frequency locking of THz photomixing with distributed-Bragg-reflector diode lasers in low-temperature-grown GaAs," Appl. Phys. Lett., vol. 71, pp. 1601-1603, 1997.

[8] S. Matsuura, M. Tani, H. Abe, K. Sakai, H. Ozeki, and S. Saito, "High resolution $\mathrm{THz}$ spectroscopy by a compact radiation source based on photomixing with diode lasers in a photoconductive antenna," J. Mol. Spectrosc., vol. 187, pp. 97-101, 1998.

[9] K. G. Libbrecht and J. L. Hall, "A low-noise high-speed diode laser current controller,” Rev. Sci. Instrum., vol. 64, pp. 2133-2135, 1993.

[10] R. W. Tkach and A. R. Chraplyvy, "Regimes of feedback effects in 1.5 $\mu \mathrm{m}$ distributed feedback lasers," J. Lightwave Technol., vol. LT-4, pp. 1655-1661, Nov. 1986.

[11] R. V. Pound, "Electronic frequency stabilization of microwave oscillators," Rev. Sci. Instrum., vol. 17, pp. 490-505, 1946.

[12] R. W. P. Drever, J. L. Hall, F. V. Kowalski, J. Hough, G. M. Ford, A. J. Munley, and H. Ward, "Laser phase and frequency stabilization using an optical resonator," Appl. Phys. B, Photophys. Laser Chem., vol. 31, pp. $97-105,1983$

[13] L. Hollberg, V. L. Velichansky, C. S. Weimer, and R. W. Fox, "Highaccuracy spectroscopy with semiconductor lasers: Application to laserfrequency stabilization," in Frequency Control of Semiconductor Lasers, M. Ohtsu, Ed. New York: Wiley, 1996, pp. 73-93.

[14] S. Matsuura, P. Chen, G. A. Blake, J. C. Pearson, and H. M. Pickett, "Simultaneous amplification of terahertz difference frequencies by an injection-seeded semiconductor laser amplifier at $850 \mathrm{~nm}$," Int. J. Infrared Millim. Waves, vol. 19, pp. 849-858, 1998

[15] D. Wandt, M. Laschek, F. v. Alvensleben, A. Tünnermann, and H. Welling, "Continuously tunable $0.5 \mathrm{~W}$ single-frequency diode laser source," Opt. Commun., vol. 148, pp. 261-264, 1998.

[16] S. Verghese, K. A. McIntosh, and E. R. Brown, "Highly tunable fiber-coupled photomixers with coherent terahertz output power," IEEE Trans. Microwave Theory and Tech., vol. 45, pp. 1301-1309, Aug. 1997.

[17] S. Matsuura, G. A. Blake, R. A. Wyss, J. C. Pearson, C. Kadow, A. W Jackson, and A. C. Gossard, "A traveling-wave $\mathrm{THz}$ photomixer based on angle-tuned phase matching," Appl. Phys. Lett., 1999, to be published.

[18] H. M. Pickett, "Determination of collisional linewidths and shifts by a convolution method," Appl. Opt., vol. 19, pp. 2745-2749, 1980.

[19] R. G. De Voe, C. Fabre, K. Jungmann, J. Hoffnagle, and R. G. Brewer, "Precision optical-frequency-difference measurements," Phys. Rev. A Gen. Phys., vol. 37, pp. 1802-1805, 1988.

[20] I. Nolt, J. V. Radostitz, G. Dilonardo, K. M. Evenson, D. A. Jennings, K. R. Leopold, L. R. Zink, and A. Hinz, "Accurate rotational constants of $\mathrm{CO}, \mathrm{HCl}$, and $\mathrm{HF}-$ Spectral standards for the 0.3 to $6 \mathrm{THz}\left(10 \mathrm{~cm}^{-1}\right.$ to $200 \mathrm{~cm}^{-1}$ ) region," J. Mol. Spectrosc., vol. 125, pp. 274-287, 1987.

[21] L. Y. Lin, M. C. Wu, T. Itoh, T. A. Vang, R. E. Muller, D. L. Sivco, and A. Y. Cho, "Velocity-matched distributed photodetectors with high saturation power and large bandwidth," IEEE Photon. Technol. Lett., vol. 8, pp. 1376-1378, Oct. 1996.

[22] Y. R. Shen, Principles of Nonlinear Optics. New York: Wiley, 1984.

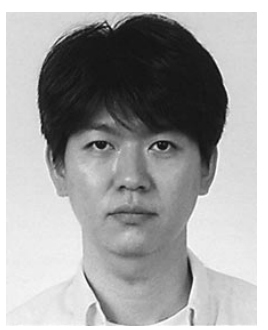

Shuji Matsuura was born in Tokushima, Japan, in 1965. He received the B.S. degree in physics from Hiroshima University, Hiroshima, Japan, in 1988, and the Ph.D. degree in physics from Nagoya University, Nagoya, Japan, in 1995. His dissertation research concerned the development of spaceborne infrared instruments and observational study of the zodiacal light and the diffuse infrared background radiation.

From 1995 to 1997, he was a COE Research Fellow at the Institute of Space and Astronautical Science, Yoshinodai, Japan, and a National Post-Doctoral Research Fellow at the Communications Research Laboratory, Kansai, Japan, where he worked on the development of terahertz sources based on the laser photomixing. From 1997 to 1999, he was a Post-Doctoral Fellow in the Division of Geologica and Planetary Sciences, California Institute of Technology, Pasadena, CA. He is currently with Infrared Astrophysics, Institute of Space and Astronautical Sciences, Kanagawa, Japan. His research interests include quantum electronics, astronomy, and development of infrared and terahertz source/detector systems.

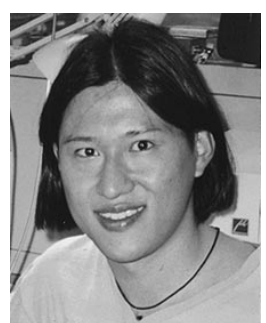

Pin Chen was born in Taipei, Taiwan, R.O.C., on May 5, 1969. He received the B.S. degree from the University of California at Berkeley, in 1991, and the Ph.D. degree in physical chemistry from the California Institute of Technology, Pasadena, in 1999.

He is currently a National Research Council Postoral Research Associate in the Time and Frequency Division, National Institute of Standards and Technology, Boulder, CO.

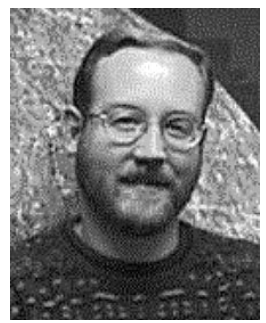

Geoffrey A. Blake was born in Greenville, MI, in 1959. He received the B.S. degree in chemistry from Duke University, Durham, NC, in 1981, and the Ph.D. degree in chemical physics from the California Institute of Technology, Pasadena, in 1986. His dissertation concerned terahertz heterodyne spectroscopy of interstellar molecular clouds and the development of laboratory terahertz spectrometers.

He then moved to the University of California at Berkeley, as a Miller Fellow, where he carried out astronomical research in star formation and the terahertz spectroscopy of hydrogen bonded clusters. In 1988, after joining the $\mathrm{Di}$ vision of Geological and Planetary Sciences, California Institute of Technology, he continued research in the observational characterization of stellar and planetary genesis, and began new programs in the development of laboratory spectroscopies at terahertz, infrared, and optical wavelengths. He is currently a Professor of Cosmochemistry and planetary science at the California Institute of Technology. His major interests include molecular astrophysics, the laboratory characterization of hydrogen bonded clusters and other reactive species, and the development of new tools to characterize the global cycling of biogenic trace gases. He has published over 100 technical papers and holds patents in the field of optical parametric oscillator devices.

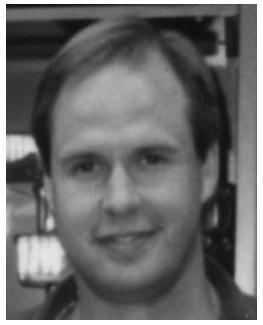

John C. Pearson received the A.B. degree for his work on microwave spectroscopy of $\mathrm{CnH}$ radicals from Harvard University, Cambridge, MA, in 1988, and the M.A. and Ph.D degrees from Duke University, Durham, NC, in 1990 and 1995, respectively, with a thesis on spectroscopic and collisional studies of molecules of astrophysical interest.

He was a Post-Doctoral Scholar at the Jet Propulsion Laboratory, California Institute of Technology, Pasadena, with research interests in the area of microwave spectroscopy and radio astronomy. Since 1997, he has been a Scientist with the Jet Propulsion Laboratory and the Instrument Manager for the heterodyne instrument for the Far Infrared and Submillimeter Telescope Mission.

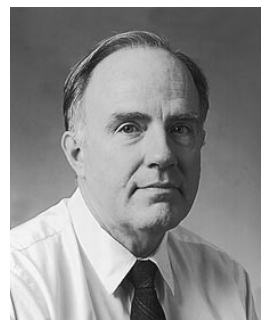

Herbert M. Pickett (M'83) was born in Baltimore, MD, on April 2, 1943. He received the A.B. degree from Williams College, Williamstown, MA, in 1965 , and the Ph.D. degree in physical chemistry from the University of California at Berkeley, in 1970.

From 1970 to 1973, he was a Post-Doctoral Fellow at Harvard University, Cambridge, MA. In 1977, he was a Miller Fellow at the University of California at Berkeley. From 1973 to 1978, he was an Assistant Professor at the University of Texas at Austin. He is currently a Senior Research Scientist and Leader of the Molecular Spectroscopy Group, Jet Propulsion Laboratory, California Institute of Technology, Pasadena. 